\title{
Efficient Cluster Head Selection Strategy With All Pair Shortest Routing In Networks
}

\author{
Mr. Anto Premkumar ${ }^{\# 1}$, Mr. Arnold Joseph ${ }^{\# 2}$, Mr. Kaushik Moses ${ }^{\# 3}$ \\ Mr. Anil P chacko \#4 \\ Assistant Professor-ECE $E^{\# 1}$, Student $-E C E^{\# 2}$, Student-ECE $E^{\# 3}$, Student-ECE ${ }^{\# 4}$, \\ CSI College of Engineering -The Nilgiris
}

\begin{abstract}
Clustering is an effective way for prolonging the lifetime of a wireless sensor network. Clustering in the network consumes much time in organizing the nodes and they hardly consider the energy consumption problem in forming the cluster and in selecting the cluster heads among the other nodes.In this work, an efficient cluster head selection scheme is organized with inculcating the all pair shortest path stratergy which inturn reduce the consumption of energy in the network and increase the life time of the network even in the dynamic network mode. By this formulae, it has been viewed that amont of time taken to send the packet to the destination and the energy consumed by the networks is partially reduced and the network lifetime is improved.
\end{abstract}

Keywords: Cluster head, All pair shortest path.

\section{Introduction}

Wireless sensor networks(WSNs) are composed of large number of low cost and tiny nodes which has the capability of sensing the environment arount it. Such as military surveillance, tracking, environment monitoring, forecasting and health care etc., these cheap sensors are deployed in the physical environment to be monitored and they transmit, collect datas whict cannot be attended any more.

Energy efficiency and sending the packet in a minimum time with least bandwidth is a primary challenge in WSNs. Since the sensor nodes are powered with limited batteries and cannot be recharged once deployed there should be a proper scheme to enhance the network lifetime. Many clustering algorithms are developed for cluster formation, cluster head selection and to send the packet in a best path where only the parameters are limited to energy, delay and so on. Which inturn increase the network lifetime. Hop number \& hop distance are the another important parameters which has been taken into account for analysis. When the hop number is too large, it increases the latency in the network and reduces the energy consumption. Where in reverse, the energy consumption is more and ent to end delay decreases. So, now comes the necessicity for optimizing the hop number with suitable individual distance(s) inorder to achieve energy reduction and balancing in the network.

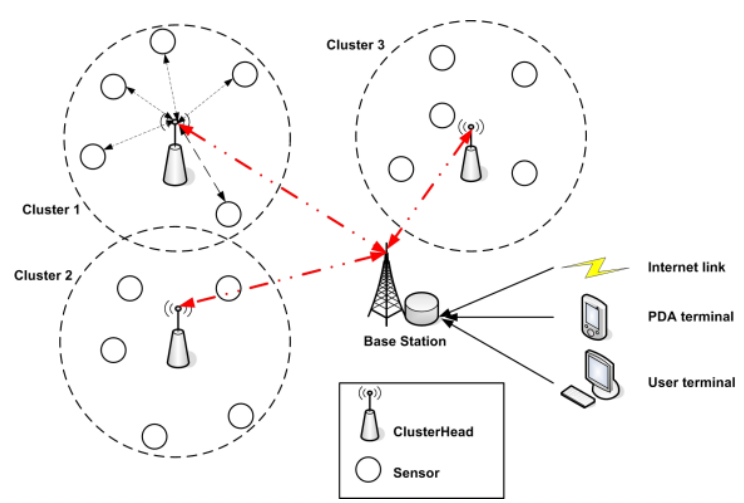

Fig 1. General sensor node structure

Following are the key features of WSNs network:

1.Increasing the battery level, paralley increasing its network lifetime.

2. Increasing the residual energy efficiency.

3. Predicting the location of the node is the practical difficulty because of large amount of over head and large number of nodes in the network.

4. All the sensor nodes that has to be discussed are application oriented, so that the parameters as outcome are narrow down. 
In this paper we present a efficient cluster head selection concept and in parallel routing the packet through all pair best path via cluster head. The outcome of the work will bring out the highlevel balancing in the network and low level energy consumption of the sensor nodes inspite of large overheads in the module.

\section{Related Works}

Wireless sensor nodes has certain constraints associated with them particularly minimizing energy consumption is the basic and key requirement in formulating the sensor network protocols. Since the nodes are small, it is unattendable, batteries may drain out very fast, so it is necessary that the network be energy efficient inorder to maximize the lifetime of the networks[1,2]. It demands fault tolerance scalability, production costs and reliability[1].By using direct diffusion(DD), a query based technique for routing the packet and by aggregating, caching and reinforcement the suitable link is dynamically selected for transmission. In LEACH protocol the selection of cluster head is elected dynamically in rotation. Data fusion is adopted to reduce the amount of data send over the link in the network[3]. In continuation the time interval is partitioned into fixed intervals with equal length and updation is made for selection of $\mathrm{CH}$ here[4,5]. Sensors sense and gathers the datas of its sensing and through the data forwarding transmission technique the datas are transmitted[5,6]. Some stochastic mode of technique is adopted for cluster head selection. In HCR method each cluster is managed with the set of associates and the lifetime of the clusters last long[7]. In S-MAC concept, some random nodes are put to sleep mode for a particular time till then the traffic is controlled by storing the packet in the neighbouring nodes. When the node is $\mathrm{ON}$ it starts retrieving the packets from its neighbouring nodes. This mode selection is made in random so that the consumption of energy by the network is maintained low[8]. In some papers it is discussed that the cluster head is selected based on the number of times the node has been a cluster head so far or by choosing the random number and if its below the optimized threashold that particular nodes is given a chance as a cluster head in the network[9]. Some cases by deducing the hierarchial clustering strategy it has been found that the energy savings increases with the number of levels in the hierarchy[10]. As a conclusion, CHs are elected based on the various techniques seen above and these strategies supports the concept of reducing the residual energy in the network. Even the concept of iteration plays a vital role in electing the heads as term basis and performs the task. Critical datas are send out to the destination immediately after sensing the status of it.

\section{The Proposed Algorithm}

In this section, we describe our proposed algorithm in detail. We first introduce the network model, and then describe the performance analysis of the above designed network.

\section{A.Network Model}

In this model few sensor nodes are deployed in a particular arena say 1200X1200 sq.m. The below network is dynamically constructed. The Distance in all pair paths are deduced in all the modes every second so that the technique is made efficiently. All the nodes are arranged as a cluster in the arena based on the criterias of the algorithm.

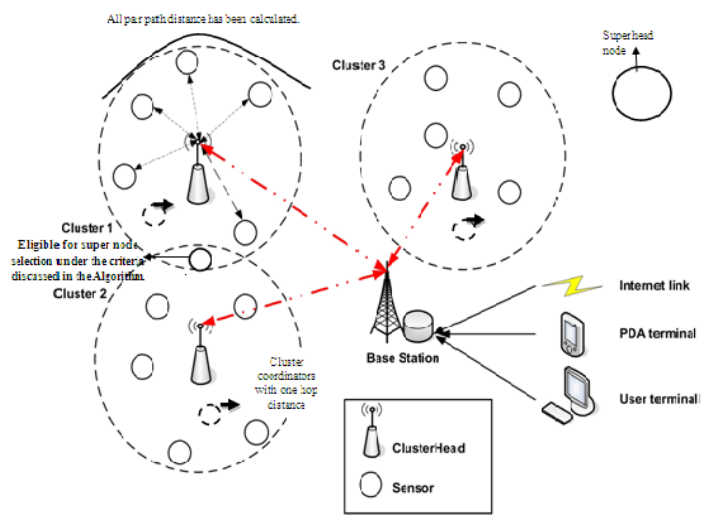

Fig2. Network model of DECHA 


\section{B. Mathematical analysis}

Proposed formulae:

$$
\mathrm{P} * \mathrm{D}_{\text {avg-inter }} * \mathrm{~N}_{\text {max_energy }}
$$

$1-\mathrm{P}\left(\mathrm{n} \bmod \mathrm{P}^{\mathrm{n}-1}\right) * \mathrm{~N}_{\text {current }}{ }^{*} \sum \mathrm{D}_{\text {inter_node }} * \mathrm{D}_{\mathrm{CH}}$ from center_avg

Where

Criterias:

$\mathrm{D}_{\text {avg-inter }}$ average distance between the cluster nodes-all pair distance

$\mathrm{N}_{\text {max_energy }}$ Maximum energy node

$\mathrm{N}_{\text {current }}$ current energy of the node

$\sum \mathrm{D}_{\text {inter_node }}$ Sum of all pair path distance

$\mathrm{D}_{\mathrm{CH} \text { from center_avg }} \mathrm{CHs}$ distance from the other member nodes.

\section{C.Proposed Algorithm}

Pseudo code for the proposed algorithm to select the cluster head among the sensor nodes .This false code putforth the hint to develop the arena.

Algorithm: Proposed algorithm

Set up ()

Sensor nodes send information about its existence to the base station through the beacon signal every second.

Geographical group the data

Determining the good clusters

In each cluster, all sensor nodes send energy level and distance between the nodes (each other)

\section{CH Selection}

If sensor node with highest energy level and shortest distance nodes then Choose $\mathrm{CH}$ and uncast the information of the member node (at least 5 clusters for 50 nodes)

Else

Choose nodes as member node

End if

Nodes send data to $\mathrm{CH}$ (transmitter uses a minimal energy) and minimal distance i.e. all pair best path The power of each non $\mathrm{CH}$ can be turned off until the nodes allocated transmission time.

$\mathrm{CH}$ performs data aggregation.

Aggregated data is sent to BS (transmission takes high energy and minimal distance) i.e. all pair best path.

Residual energy on decrease

If (CN's residual energy $<$ threshold level)

If (CH's all pair path $>$ coverage area distance)

$\mathrm{CH}$ is re elected -Go to step 6

Mean while selecting the shortest distance from the nodes to form the cluster head

\section{Cluster head coordinator}

If Anchor node in each cluster, which will gather all the information about that cluster then The Anchor node is also known as cluster coordinator Cluster coordinator has more knowledge about the cluster and the arena (one hop node with high energy) The gathered energy will send to the cluster head with one hope distance and minimal bandwidth.

\section{Super node selection}

Cluster nodes are not stationary they keep moving when they tend to intersect other node, super node will be formed.

It is common for all other cluster nodes

It reduces all pair path and energy efficiency will be maximum.

The same set of nodes which are inter selected and is collectively known as Super node. 


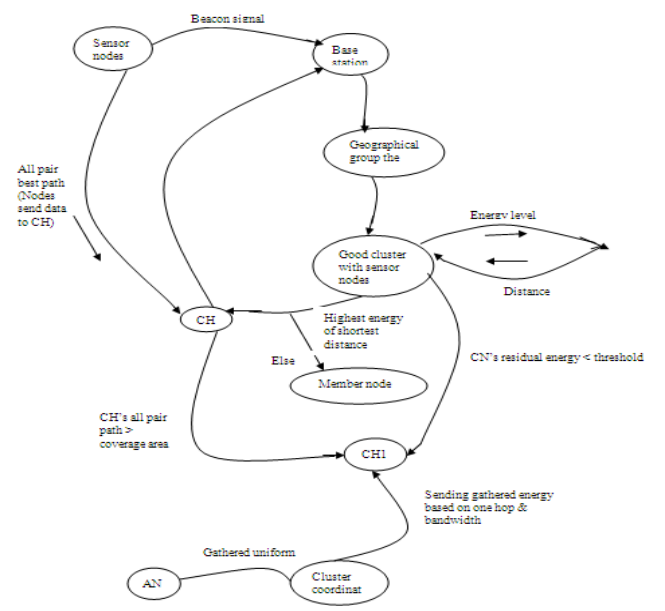

Fig.3. Transistion Diagram for Proposed Algorithm

To construct the arena, few nodes have been deployed in the field where least mobility is given to the sensor nodes. All the nodes register their existence in the nearer base station and update the same.Geographically the cluster boundaries has been destained. Residual energies and all pair path distance have been calculated in the network. The node that has higher residual energy and lower distance among the boundary is selected as cluster head oters acts as a members of it. The one hop distance node from the CHs is considered as cluster co-ordinator which has the knowledge of the arena. Through this $\mathrm{CHCs}$ (cluster coordinator) only the datas are send at a shorter distance effectively. Due to its mobility the nodes tend to move away from the clustion where without delay re-election have to be conducted for CHs selection. The nodes which moves away the cluster and comes in intersection of the other boundary and by comparing its energy efficiency and shortest distance withrespect to the others the eligibility to elect as a super cluster node. The routing is done between the $\mathrm{CHs}$ through the all pair shortest path in connection. As a outcome the time and energy consumption of the network is partially reduced.

\section{Performance Evaluation}

The performance evaluation on energy consumption, Throughput, delay, Network lifetime and Time taken for cluster formation was executed and compared with the existing techniques.

Table I Experimental Scenario

\begin{tabular}{|l|l|}
\hline Number of nodes & $250-400$ \\
\hline Initial Energy & 1000 joules \\
\hline Wireless Range & Infinity \\
\hline Buffer & 16 packets \\
\hline
\end{tabular}

TABLE II EXISTING VS. PROPOSED SYSTEMS

\begin{tabular}{|c|c|c|c|}
\hline PARAMIETERS & & STING & PROPOSED \\
\hline \multirow{5}{*}{ ENERGY CONSCMIPTION } & LEACH & $15 \%$ & \multirow{5}{*}{$12 \%$} \\
\hline & TL-LEACH & $35 \%$ & \\
\hline & EECS & $85 \%$ & \\
\hline & PEGASIS & $20 \%$ & \\
\hline & GROUP & $66 \%$ & \\
\hline \multirow{5}{*}{ THROUGHPUT } & LEACH & $75 \%$ & \multirow{5}{*}{$94 \%$} \\
\hline & TL-LEACH & $64 \%$ & \\
\hline & EECS & $23 \%$ & \\
\hline & PEGASIS & $75 \%$ & \\
\hline & GROUP & $32 \%$ & \\
\hline \multirow{5}{*}{ DELAY } & LEACH & $42 \%$ & \multirow{5}{*}{$14 \%$} \\
\hline & TL-LEACH & $31 \%$ & \\
\hline & EECS & $76 \%$ & \\
\hline & PEGASIS & $42 \%$ & \\
\hline & GROUP & $80 \%$ & \\
\hline \multirow{5}{*}{ NETWORK LIFETINE } & LEACH & $80 \%$ & \multirow{5}{*}{$96 \%$} \\
\hline & TL-LEACH & $72 \%$ & \\
\hline & EECS & $20 \%$ & \\
\hline & PEGASIS & $79 \%$ & \\
\hline & GROUP & $26 \%$ & \\
\hline \multirow{5}{*}{$\begin{array}{l}\text { TINE TAKEN FOR } \\
\text { CLUSTER FORIATION }\end{array}$} & LEACH & FAIR & \multirow{5}{*}{ GOOD } \\
\hline & TL-LEACH & PARTIALY FAIR & \\
\hline & EECS & POOR & \\
\hline & PEGASIS & FAIR & \\
\hline & GROUP & POOR & \\
\hline
\end{tabular}




\section{E.Energy Level}

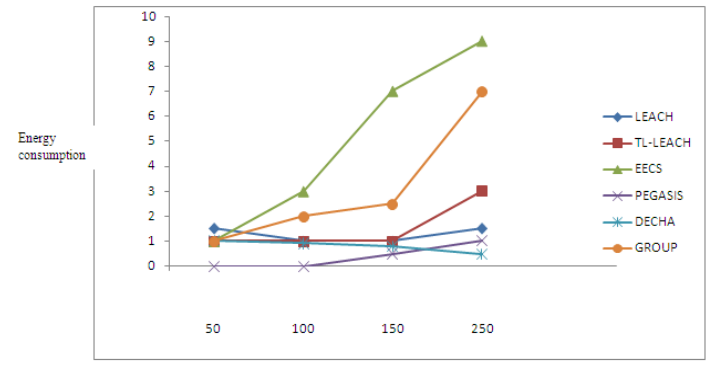

Fig 4. Energy Level

The above graph proves that the energy consumption of the cluster based network interms of DECHA is less compared to the other protocols. It reduces to about $12 \%$ of energy consumption.

\section{F.Throughput}

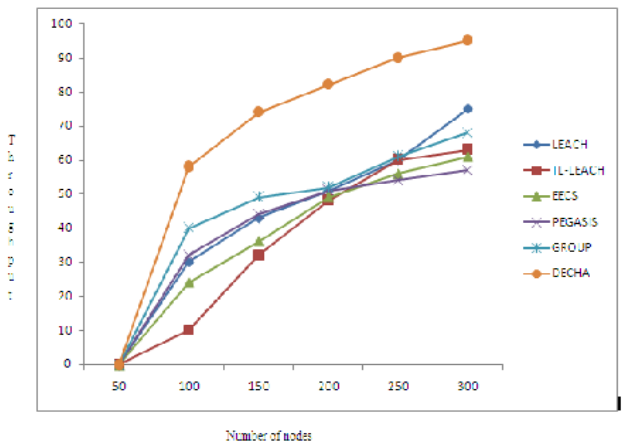

Fig 5.Throughput

Because of the reduced energy consumption and the time taken to reachout the destination, the throughput of the network is $94 \%$ which is comparatively higher than the other protocols because of the fast computation of shortest paths.

\section{G.Delay}

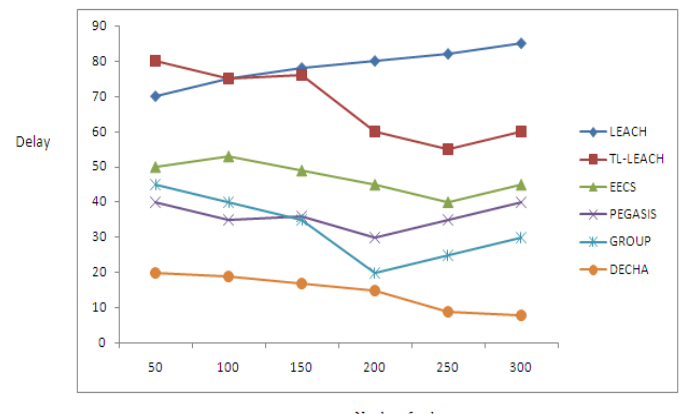

FIg.6. Delay

Delay in the proposed system is reduced partially but then calculating the all pair best path from time to time in a dynamic mode makes the delay to be little high.

\section{G.Lifetime}

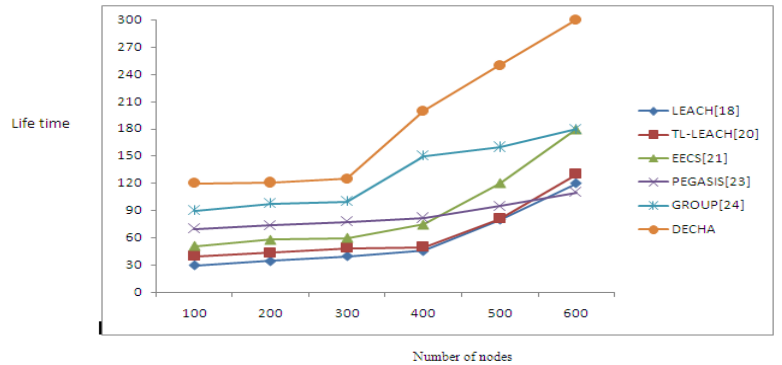

Fig 7. Network Lifetime 
With the previous statistics the energy consumption of DECHA is very low so the withstanding power of the sensors battery is more. Though the nodes are unattendable, because of its efficiency in consumption/energy saving, the entire network's lifetime have been increased.

\section{G.Time taken for cluster formation}

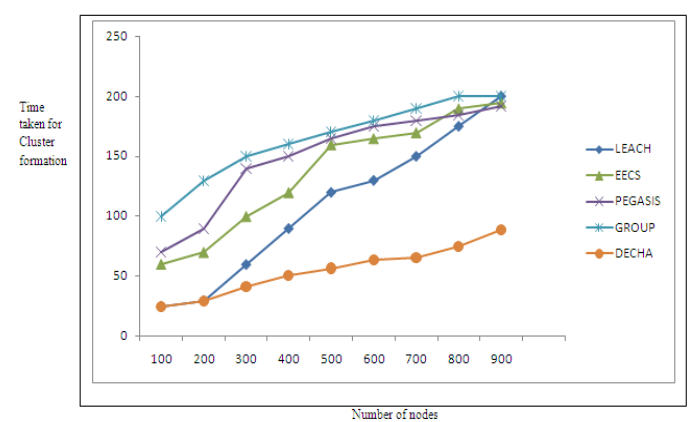

Fig 8. Time taken for cluster formation

As for as the formation of cluster in the network is concern, because of high level computational efficiency of DECHA it is understood that based on the fixed criteria it is quick and fast in formation. and our algorithms show better efficiency in terms of energy, throughput, and fast computational efficiency and the amount of energy consumed is less because of quick response.

\section{Simulation Results}

In this section, the performance of our proposed algorithm is compared using the simulated results Hence the throughput of the entire network is partially good. Because of high overheads due to large computation the delay is marginally increased. Selection of cluster heads in quick response is the efficient way to have better communication. Techniques are developed for fast recovery from single-link failures to provide more than one forwarding edge to route a packet to a destination.

\section{Conclusion And Future Work}

DECHA-Distance and Energy based Cluster head selection All pair path routing algorithm shows a better out performance in the field of energy, computation and increasing the lifetime of the network and the time taken by the packet to reach the destination. And it has been overviewed that this scheme is very suitable for wireless networks as for as energy efficiency is concerned. As a future work the research takes the direction on relying the strategy of hierarchy, mode based selection, sleep and awake mode selection especially for the dynamic topology. In future the above completed task can be modified by some other new techniques and algorithms which will reduce the delay time and traffic even more and helps to transmit the packets with high speed to reach the destination.

\section{Acknowledgement}

First and foremost we thank the Lord almighty for showering his grace and wisdom to carry out this work we extend our greatful thank to all the staffs who extend their valuble thoughts to carry out this work successfully.

\section{References}

[1] I.F.Akyilidiz et al, "Wireless Sensor Networks: A Survey," Elsevier sci.B.V.Comp.Networks, Vol.38,No.4, Mar 2002.

[2] W.B.Heinzelmann, A.P.Chandrakaran, and H. Balakrishnan,"An application-specific protocol architecture for wireless micro sensor networks," IEEE Trans.Wireless commun, Vol 1,no.4,oct 2002.

[3] C. Intanagonwiwat, R.Govindan and D.Eslrin," Directed diffusion A scalable and robust communication paradigm for sensor network" proc. $6^{\text {th }}$ Annual ACM/IEEE international conference on mobile computing and networking(Mobi com'00) Aug 2000.

[4] W. Heinzelman, A. Chandrakaran and H. Balakrishnan,"Energy efficient communication protocol for wireless micro networks" proc. Hawaii international conference system science(2000)

[5] W. Heinzelman, A. Chandrakaran and H. Balakrishnan,"An application specific protocol architecture for wireless microsensor networks" IEEE Trans. On wireless commun.,-2002.

[6] Xiaoli Li, Hongchi Shi, and Yi Shang," A Sorted RSSI Quantization Based Algorithm for Sensor Network" Transactions on Mobile Computing,Vol. 6, Issue 11, pp. 1284-1296, 2007.

[7] S. Olariu and I. Stojmenovic, Design guidelines for maximizing lifetime and avoiding energy holes in sensor networks with uniform distribution and uniform reporting, in: Proceedings of IEEE INFOCOM (April 2006).

[8] O. Younis and S. Fahmy, HEED: a hybrid, energy-efficient, distributedclustering approach for ad hoc sensor networks, IEEE Transactions on Mobile Computing 3(4) (2004) 660-669.

[9] S. Lindsey and C. Raghavendra, "PEGASIS: power-efficient gathering in sensor information S. in Proc. of IEEE Aerospace Conference, vol. 3, pp.1125-1130, March 9-16, 2002.Article (CrossRef Link) 
[10] Deng, J. Multi-hop/Direct Forwarding (MDF) for static wireless sensor networks. ACM Trans. Sens. Netw. 2009, 5, 1-25.

[11] Wang, J.; Cho, J; Lee, S.; Chen, K.C.; Lee, Y.K. Hop-based Energy aware routing algorithm for wireless sensor networks. IEICE Trans. Commun. 2010, 2, 305-316.

[12] C. F. Chiasserini, I. Chlamtac, P. Monti, and A. Nucci, "Energy Efficient Design of Wireless Ad Hoc Networks," Proceedings of European Wireless, Feb 2002.

[13] S. Bandyopadhyay and E. J. Coyle, “An Energy Efficient Hierarchical Clustering Algorithm for Wireless Sensor Networks," IEEE INFOCOM, April 2003

[14] D. J. Baker and A. Epheremides, “The Architectural Organization of a Moblie Radio Network via a Distributed Algorithm," IEEE Transactions on Communications, vol. Com-29, no. 11, November 1981.

[15] P. Tsigas, "Project on Moblie Ad Hoc Networking and Clustering for the Course EDA390 Computer Communcation and Distributed Systems," Manual for University Course.

[16] A. Amis, R. Prakash, T. Vuong, and D. Huynh, "Max-Min D-Cluster Formation in Wireless Ad Hoc Networks," IEEE INFOCOM, March 2000.

[17] C.E.Nishimura and D.M.Conlon, "IUSS dual use: Monitoring of whales and earthquakes using SOSUS," Mar. Technol. Soc. J., vol. 27, no. 4, 1994.

[18] A. Mainwaring et al., "Wireless Sensor Networks for Habitat Monitoring," Proceedings of the 1st ACM International Workshop on WSN, 2002.

[19] C.Y.Chong, S.Mori, and K.C.Chang, "Distributed multitarget multisensory tracking," in Multitarget Multisensor Tracking:Advanced Applications, 1990.

[20] C. Intanagonwiwat et al., "Directed Diffusion for Wireless Sensor Networking," IEEE/ACM Transaction on Networking, vol. 11, no. 1 , Feb. 2003

[21] M. Chatterjee, S. K. Das, and D. Turgut, "WCA: A Weighted Clustering Algorithm for Mobile Ad Hoc Networks," Clustering Computing, vol. 5, pp. 193-204, 2002.

[22] W. C. Y. Lee, "Overview of Cellular CDMA," IEEE Trans. on Vehicular Technology, vol. 40, no. 2, pp. 291-302, May 1991.

[23] W. R. Heinzelman, A. Chandrakasan, and H. Balakrishnan, "Energy- Efficient Communication Protocol for Wireless Sensor Networks," Proceedings of the 33th Hawaii International Conference on System Sciences-2000.

[24] W. Choi, P. Shah, and S. K. Das, "A Framework for Energy-Saving Data Gathering Using Two-Phase Clustering in Wireless Sensor Networks", in Proceedings of Int'l Conference on Mobile and Ubiquitous Systems: Networking and Services (MOBIQUITOUS), 2004.

[25] W. Heinzelman, A. Chandrakasan, and H. Balakrishnan, "An Application-Specific Protocol Architecture for Wireless Microsensor 2002.

[26] O. Younis and S. Fahmy, "HEED: A Hybrid, Energy-Efficient, Distributed Clustering Approach for Ad Hoc Sensor Networks", IEEE Transactions on Mobile Computing, vol. 3, no. 4, 2004.

[27] S. Lindsey, C. Raghavendra, K. M. Sivalingam, "Data Gathering Algorithms in Sensor Networks Using Energy Metrics", IEEE Transactions on Parallel and Distributed Systems, vol. 13, no. 9, 2002.

[28] S . Soro and W. Heinzelman, "Prolonging the Lifetime of Wireless Sensor Networks via Unequal Clustering", in Proceedings of the 19th IEEE International Parallel and Distributed Processing Symposium (IPDPS),2005.

[29] M. Ye, C. F. Li, G. H. Chen, and J. Wu, "EECS: An Energy Efficient Clustering Scheme in Wireless Sensor Networks", in Proceedings of IEEE Int'l Performance Computing and Communications Conference (IPCCC), 2005.

[30] Wiley Series on Wireless Communications and Mobile Computing FUNDAMENTALS OFWIRELESS SENSORNETWORKSpublished in 2010 